\title{
Relações empíricas entre a estrutura da vegetação e dados do sensor TM/LANDSAT ${ }^{1}$
}

\author{
Luciano J. de O. Accioly², Admilson Pachêco ${ }^{3}$, Thomaz C. e C. da Costa ${ }^{4}$, \\ Osvaldo F. Lopes ${ }^{2} \&$ Maria A. J. de Oliveira ${ }^{5}$
}

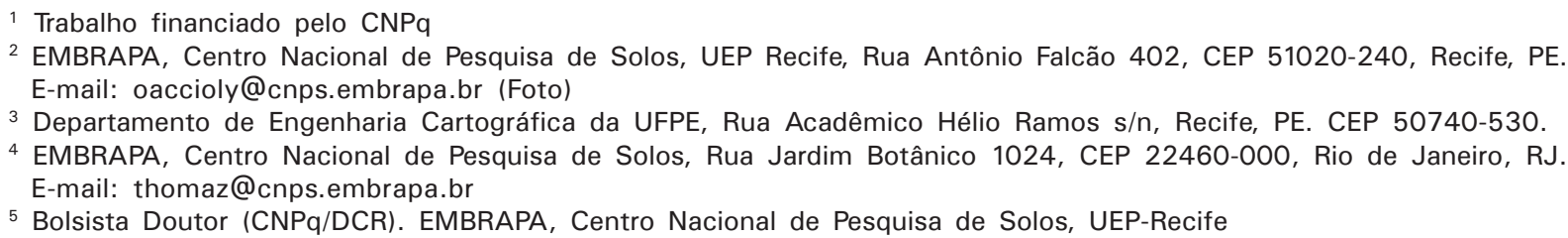

Protocolo $75-28 / 5 / 2001$

\begin{abstract}
Resumo: A utilização de dados espectrais, na estimativa de parâmetros estruturais da vegetação, constitui-se num dos mais importantes papéis do sensoriamento remoto dos ecossistemas naturais. Neste trabalho foram aplicadas transformações espectrais, como os índices de vegetação e a fração de vegetação obtida pelo modelo de análise de misturas em dados do sensor TM/LANDSAT, com o objetivo de se estimar parâmetros estruturais da Floresta Nacional do Araripe, no Ceará. As imagens foram calibradas para radiância, ao nível do solo, e antes do processamento foram removidos seus "outliers". Tanto a fração de vegetação verde quanto os índices de vegetação e as respostas de bandas individuais, apresentaram correlação positiva e significativa com as estimativas da altura média da parcela, da área basal à altura do peito e da área basal na base. Os valores de coeficientes de correlação variaram entre 0,33 e 0,60. Os melhores relacionamentos foram obtidos com o índice de vegetação da razão simples e com o índice estrutural da vegetação para os quais foram estabelecidas as equações de regressão.
\end{abstract}

Palavras-chave: sensoriamento remoto, análise de misturas espectrais, índices de vegetação, variáveis estruturais, tipologias de vegetação

\section{Empirical relationship between vegetation structure and TM/LANDSAT data}

\begin{abstract}
The use of spectral data to estimate the structural parameters of vegetation has been considered as one of the most important applications of the remote sensing of ecosystems. In this paper, spectral enhancements and transformations such as vegetation indices, vegetation fraction from mixture analysis from TM/LANDSAT data were used to estimate structural parameters of 'Floresta Nacional do Araripe', in the State of Ceará. The image was calibrated at ground radiance and had the outliers removed before any further processing. Green vegetation fraction as well as vegetation indices and the spectral response of individual bands were all positively and significantly correlated with estimates of the mean height of the sample plot, basal area at the breast height, and basal area at the ground. The correlation coefficients varied from 0.33 to 0.60 . The best linear relationships obtained by regression between simple ratio and the structural indices of the vegetation are presented.
\end{abstract}

Key words: remote sensing, spectral mixture analysis, vegetation indices, vegetation structural variables, types of vegetation

\section{INTRODUÇÃO}

A Chapada do Araripe é caracterizada pela diversidade de tipologias da vegetação, encontrando-se áreas cobertas por carrasco, cerrado, cerradão e mata úmida. A exploração dessa vegetação vem-se processando na região por um período de aproximadamente 60 anos, principalmente na vertente sul da Chapada. As principais causas dessa devastação são as crescentes demandas por lenha para o uso industrial (indústria de gesso) e para o consumo doméstico e, por terra, para lavouras de subsistência e para o estabelecimento da pecuária. Nesse contexto, a Floresta Nacional do Araripe (FLONA) representa um dos poucos fragmentos da vegetação que originalmente dominaram a Chapada do Araripe. 
As imagens de satélite têm sido utilizadas para avaliar o estágio de crescimento e identificar a composição arbórea de florestas diversas (Franklin, 1986; Cibula, 1987). Várias técnicas de processamento de imagens são utilizadas para a extração de informações sobre tipos e parâmetros de vegetação, dentre as quais se destacam os índices de vegetação (Vis) (Huete, 1989) a classificação de imagens e a decomposição de dados espectrais, como a análise de misturas espectrais (AME).

Excetuando-se algumas transformações no espaço multiespectral, os índices de vegetação geralmente usam as respostas espectrais que os alvos apresentam no infravermelho próximo e no vermelho. Em estudos comparativos de diversos índices de vegetação em escala global, Huete et al. (1997) encontraram que o Índice de Vegetação da Diferença Normalizada (NDVI) saturava sob condições de floresta densa, enquanto sob condições de vegetação esparsa de regiões semi-áridas, o NDVI foi mais sensível ao "background" que o índice de vegetação resistente aos efeitos da atmosfera e ajustado para os efeitos de solo (SARVI). Huete et al. (1997) concluem, no entanto, que, para efeito de monitoramento, esses índices se complementam, uma vez que o NDVI se mostrou mais sensível às variações na fração da energia fotossinteticamente ativa que é absorvida pelas plantas (fAPAR) enquanto o SARVI se mostrou mais sensível aos parâmetros relacionados à estrutura do dossel das plantas. Por sua vez, Cohen \& Spies (1992) trabalhando numa escala menor e envolvendo apenas vegetação de coníferas, encontraram melhor relacionamento entre parâmetros estruturais da vegetação e os índices de umidade ou "wetness" (WI) da transformação do 'Tasseled Cap'. Ao estudarem os efeitos de parâmetros do solo, como a textura e a umidade, Todd \& Hoffer (1998) concluíram que, para um mesmo percentual de cobertura, o índice de verdor (GVI) foi menos afetado pelo 'background' solo que o NDVI.

Em florestas de Pinus, Jakubauskas \& Price (1997) obtiveram um coeficiente de determinação $\left(R^{2}\right)$ de 0,62 com o índice de área foliar (IAF) em função do NDVI e do GVI, e um $\mathrm{R}^{2}=0,59$ para biomassa em função do NDVI.

Com o objetivo de reduzir a influência de componentes, como solo e outros, que não representam vegetação, novos modelos de transformações espectrais foram introduzidos para ressaltar a contribuição da vegetação. Entre esses modelos se encontra a análise de misturas espectrais (AME). Com a AME, a resposta espectral de um pixel pode ser representada como o somatório dos produtos da fração da área do pixel ocupada por um componente (vegetação, solo etc.) pela resposta espectral do respectivo componente 'puro'. Smith et al. (1990) verificaram que as frações de vegetação provenientes da AME, aplicadas a imagens do sensor TM, apresentaram resultados consistentes com as informações de percentagem de cobertura verde obtidas sob condições de campo.

Dada a importância ecológica dos fragmentos de vegetação existentes na Chapada do Araripe objetivou-se, com este trabalho avaliar a relação entre informações espectrais (bandas individuais e transformações, como índices de vegetação e AME) extraídas do sensor TM/LANDSAT, e características estruturais do fragmento de floresta localizado na FLONA do Araripe.

\section{MATERIAL E MÉTODOS}

A FLONA do Araripe foi criada em 1946 sendo, pois, oficialmente, a primeira floresta nacional. A FLONA ocupa uma área de 38.263 ha, situada ao norte do setor oriental da Chapada do Araripe ( $7^{\circ} 20^{\prime} \mathrm{S} ; 33^{\circ} 27^{\prime} \mathrm{W} ; 900 \mathrm{~m}$ ), e compreende parte dos municípios do Crato, Barbalha, Santana do Cariri e Jardim, todos no Ceará (Fig. 1).

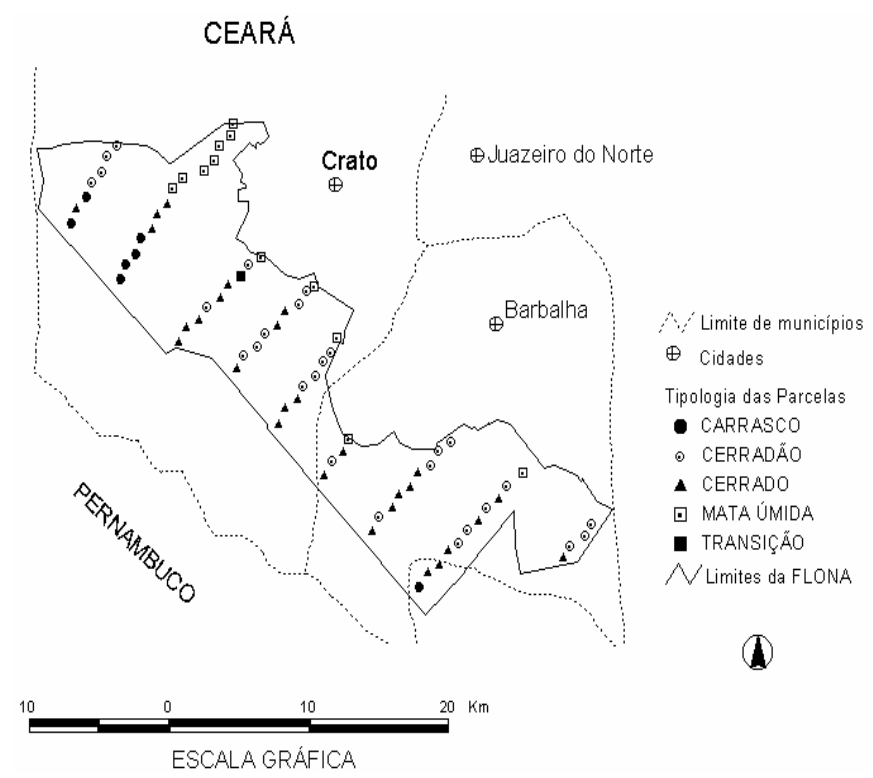

Figura 1. Localização da Floresta Nacional (FLONA) do Araripe e das parcelas amostradas por Toniolo \& Kazmierczak (1998)

A vegetação nativa cobre, praticamente, a totalidade da área da FLONA e é representada por cerca de $42 \%$ de cerrado, $37 \%$ de cerradão, $12 \%$ de mata úmida e $7 \%$ de carrasco (Toniolo $\&$ Kazmierczak, 1998). A mata úmida ou serrana, é formada por uma vegetação de porte elevado ( 8 a $15 \mathrm{~m}$ ), com mais de 100 espécies e volume de madeira da ordem de $226,22 \mathrm{~m}^{3} \mathrm{ha}^{-1}$, semelhantes à da Mata Atlântica (Campelo et al., 2000).

O cerradão apresenta volumetria semelhante à da mata úmida e caracteriza-se por apresentar árvores tortuosas, mas com densidade elevada de indivíduos e porte arbóreo, enquanto o cerrado se caracteriza pela presença de gramíneas, além de árvores e arbustos ralos e tortuosos, com altura média de $4 \mathrm{~m} \mathrm{e}$ volumetria da ordem de $72 \mathrm{~m}^{3} \mathrm{ha}^{-1}$; sua ocorrência é delimitada pelo cerradão e pelo carrasco. Este último é considerado fruto de outras formações e composto por 54 espécies, na sua maioria arbustivas e xerófitas, com altura média de $3,6 \mathrm{~m}$ e volumetria de $36,74 \mathrm{~m}^{3}$ ha $^{-1}$ (Campello et al., 2000). O relevo da FLONA apresenta-se tabular, com altitudes variando entre 760 e $960 \mathrm{~m}$. Uma vantagem desse tipo de relevo é que os seus efeitos sobre a resposta espectral da vegetação são consideravelmente reduzidos (Jakubauskas \& Price, 1997). Os solos são bem desenvolvidos e a classe dominante é a dos Latossolos, sendo o Latossolo Amarelo distrófico típico textura argilosa, a classe que mais ocorre na área. A precipitação pluviométrica média é de $1100 \mathrm{~mm}$, as temperaturas variam de 15 a $31^{\circ} \mathrm{C}$, com média em torno de $23{ }^{\circ} \mathrm{C}$ e a umidade relativa do ar varia entre $32 \mathrm{e}$ $92 \%$, com média de 62\% (Lopes \& Silva, 1998). 
A imagem utilizada nesse trabalho corresponde a um segmento de cena do TM/LANDSAT (cena 217/65) com data de passagem em 2/11/1994, e foi registrada e georreferenciada ao sistema de projeção UTM, tomando-se como referência mapas básicos planialtimétricos, na escala de 1:100.000, e os resultados conferidos com alguns pontos selecionados para verificação, "in loco", com auxílio de um receptor GPS. O erro no registro das imagens foi de 0,75 pixel.

Os valores de níveis de cinza (NC) foram convertidos para radiância, utilizando-se os coeficientes de calibração apresentados por Thome et al. (1997). A correção atmosférica se deu por meio do método dos alvos escuros aperfeiçoado, conforme Chavez (1996). A previsão de utilização dos dados do sensor TM para monitoramento justificou a conversão de NC para radiâncias ao nível do solo. Posteriormente, as imagens convertidas para radiância foram realçadas por meio de dois processamentos básicos: a) da aplicação da análise de misturas espectrais (AME) e b) da aplicação de diferentes índices de vegetação.

A AME assume que a resposta espectral de um pixel na banda c $(\mathrm{Pc})$ corresponde ao somatório das contribuições espectrais dos componentes individuais (membros finais) presentes neste pixel:

$$
\mathrm{P}_{\mathrm{c}}=\sum_{\mathrm{i}=1}^{\mathrm{N}} \mathrm{F}_{\mathrm{i}} \mathrm{R}_{\mathrm{i}, \mathrm{c}}+\mathrm{E}_{\mathrm{c}}
$$

em que:

Fi - fração do membro final i

Ri,c - resposta espectral do membro final i na banda c

Ec - erro no ajuste do modelo na banda c para um número $\mathrm{N}$ de membros finais

As imposições do modelo são as de que o número de membros finais tem que ser igual ou menor que $\mathrm{N}$ e que

$$
\sum_{i=1}^{N} F_{i}=1
$$

A AME foi considerada bem sucedida quando o erro médio para todas as bandas foi inferior a $10 \%$.

Para a aplicação da AME, as imagens foram convertidas em arquivo do tipo ASCII e importadas pelo software S-Plus, para análise estatística de dados. Os dados (valores em radiância) desses arquivos foram, então, testados quanto à normalidade em cada banda, teste necessário na medida em que a normalidade dos dados é uma premissa básica para a aplicação da AME. Foram considerados 'outliers' os pixels com radiância superior ou inferior aos valores contidos no intervalo definido pela diferença entre o terceiro e o primeiro quartis (Mathsoft, 1997). Estão associadas aos 'outliers' feições que, no campo, correspondem a áreas com algum tipo de intervenção humana. Áreas com vegetação degradada pelo pastoreio e/ou fogo, áreas ocupadas por construções diversas, entre outras, são exemplos de feições associadas aos 'outliers'. Esses 'outliers' foram removidos da imagem nos processamentos subseqüen- tes. A dimensionalidade dos dados foi testada, inicialmente, por meio da reconstrução da imagem, com base na análise fatorial (Malinowski, 1991). Esta reconstrução é uma forma prática de se determinar o número de membros finais a ser incluído na Eq. (1), feita passo a passo incluindo-se, de início, o primeiro autovetor; em seguida, o primeiro e o segundo autovetores, e assim sucessivamente. Em cada passo da reconstrução, o valor de Ec é avaliado e o número de membros finais é definido quando se alcança o valor desejado para Ec. Neste caso, um valor médio igual ou inferior a $10 \%$ para Ec, em cada banda, foi adotado como critério para se achar a dimensionalidade dos dados. Para extração de pixels "puros", utilizou-se a técnica do diagrama de dispersão, sendo considerado, principalmente, o comportamento espectral nas bandas 3 (vermelho) e 4 (infra-vermelho próximo) do sensor TM.

Os seguintes índices de vegetação foram testados: Índice de Vegetação da Diferença Normalizada (NDVI); Índice de Vegetação Ajustado para os Efeitos do Solo (SAVI); Índice de Vegetação da Razão Simples (SR); Índice Estrutural da Vegetação (SI); Índice Perpendicular de Vegetação (PVI) e Índice de Verdor (GVI)-

Para os dados do sensor TM, as equações que definem esses índices são:

$$
\text { NDVI }=(\text { TM4 }- \text { TM3) } /(\text { TM4 }+ \text { TM3 })
$$

$$
\mathrm{SR}=\mathrm{TM} 4 / \mathrm{TM} 3
$$

$$
\mathrm{SI}=\mathrm{TM} 4 / \mathrm{TM} 5
$$

$$
\begin{aligned}
\mathrm{PVI} & =\left[(\mathrm{TM} 3 \text { solo }-\mathrm{TM} 3 \text { pixel })^{2}+(\text { TM4solo }\right. \\
& \left.-\mathrm{TM} 4 \text { pixel })^{2}\right]^{1 / 2}
\end{aligned}
$$

$$
\begin{aligned}
\mathrm{GVI} & =-0,2728 \mathrm{TM} 1-0,2174 \mathrm{TM} 2-0,5508 \mathrm{TM} 3+0,7221 \mathrm{TM} 4 \\
& +0,0733 \mathrm{TM} 5-0,1648 \mathrm{TM} 7-0,7310
\end{aligned}
$$

$$
\mathrm{SAVI}=[(\mathrm{TM} 4-\mathrm{TM} 3) /(\mathrm{TM} 4+\mathrm{TM} 3+\mathrm{L})](1+\mathrm{L})
$$

Nas equações acima, TM1 a TM7 correspondem às bandas de 1 a 7 do sensor TM .

Os índices NDVI, SR e SI são tipicamente relacionais. O PVI e o GVI são índices de vegetação que usam combinação linear das respostas espectrais, enquanto o SAVI é um índice de vegetação que tenta tirar vantagens das propriedades dos índices relacionais e ortogonais, de forma a minimizar os efeitos do solo como background (Huete, 1989). No cálculo do PVI, os valores extraídos com base na linha de solo para TM3solo e TM4solo na Eq. 5 foram, respectivamente, $29,31 \mathrm{e} 32,25 \mathrm{~W} \mathrm{~m}^{2} \mathrm{Sr}^{-1} \mu \mathrm{m}^{-1}$.

O GVI representa, essencialmente, o contraste entre as bandas 1,2 e 3 (azul, verde e vermelho, respectivamente) e a banda 4 (infra-vermelho próximo). Os coeficientes para o cálculo do GVI (assim como para os demais índices da transformação do 'Tasseled Cap') para o sensor TM, têm sido incorporados a alguns softwares de processamento de imagem. Neste trabalho foram utilizados, para o cálculo do GVI, os coeficientes disponíveis no software ERDAS 8.3.1. Esses coeficientes coincidem com os apresentados por Crist et al. (1986), citados por Todd \& Hoffer (1998); no entanto, não apresentam o termo aditivo. 
Na Eq. 8, L varia de acordo com a cobertura de solo. Para as condições de vegetação aberta, Huete \& Warrick (1990) encontraram que 0,5 era um valor adequado para $\mathrm{L}$. Com base no trabalho de Huete \& Warrick (1990) e na ausência de dados experimentais que justificassem a adoção de outro valor adotou-se, neste trabalho, o valor 0,5 para $\mathrm{L}$.

Os resultados obtidos com as bandas individuais, a AME e os índices de vegetação citados anteriormente, foram relacionados com os dados estruturais da vegetação.

O levantamento florístico e dendrométrico da FLONA foi realizado em meados da década de 90, pelo PNUD/IBAMA (Toniolo \& Kazmierczak, 1998) e os dados foram gentilmente cedidos pelo IBAMA e pelo Departamento de Engenharia Florestal da Universidade Federal Rural de Pernambuco UFRPE, os quais foram convertidos para o formato Excel e processados neste "software" e/ou exportados para o pacote estatístico S-Plus. No trabalho do PNUD foram lançadas 76 parcelas com as dimensões de $10 \times 50 \mathrm{~m}$, que foram dispostas em transectos no sentido sudoeste/nordeste, conforme apresentado na Figura 1; em 51 delas foram avaliadas: altura média da parcela (HTMED, em m), área basal à altura do peito (ABPSOM, em $\mathrm{m}^{2} \mathrm{ha}^{-1}$ ) e área basal na base (ABBSOM, em $\mathrm{m}^{2} \mathrm{ha}^{-1}$ ). Essas variáveis foram obtidas apenas para as plantas com diâmetro à altura do peito superior a $5 \mathrm{~cm}$ e sua distribuição estatística foi avaliada e, após a eliminação dos 'outliers', restaram 49 parcelas, que foram utilizadas no corrente trabalho.

Enfim, na determinação do relacionamento entre as variáveis estruturais e as variáveis extraídas da imagem, foi usado o coeficiente de correlação. Modelos de análise de regressão linear foram ajustados para estimar os dados estruturais a partir dos dados espectrais para as variáveis que apresentaram maior correlação.

\section{RESULTADOS E DISCUSSÃO}

A análise individual das bandas do sensor TM evidenciou elevado desvio da normalidade, em particular na direção das radiâncias mais elevadas. A aplicação de um limiar ('threshold') utilizando-se os valores de radiância do primeiro e terceiro quartis implicou na redução de cerca de $9 \%$ sobre o número de pixels da imagem original da FLONA. A distribuição espacial dos 'outliers' removidos quando comparados com a imagem original, é mostrada na Figura 2B para a imagem do NDVI.

A reconstrução dos dados originais da imagem TM para a FLONA, por meio da análise fatorial, mostrou que três é a dimensão desses dados (Tabela 1). O conhecimento de campo e a visualização dos planos de informação (notadamente o que espacializa as classes de cobertura) agregados à interpretação visual das imagens, mostraram que os três membros finais pertenceriam às seguintes classes: vegetação verde, solo descoberto e sombra. O pixel referente à vegetação verde está localizado na área de ocorrência de mata úmida fechada e o pixel referente ao solo, encontra-se numa área onde, por interferência humana (pastoreio), a cobertura vegetal é das mais baixas dentro da área da FLONA. Por outro lado, o pixel associado à sombra localiza-se nas encostas da chapada e é representativo da sombra causada pelo efeito do relevo. A curva espectral para cada membro final utilizado na AME pode ser observada na Figura 3.
A.

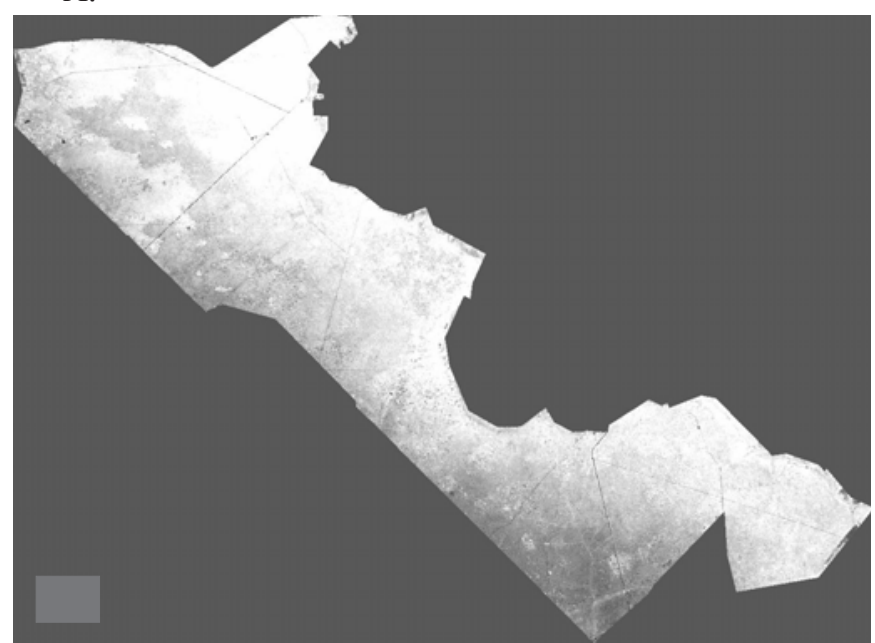

B.

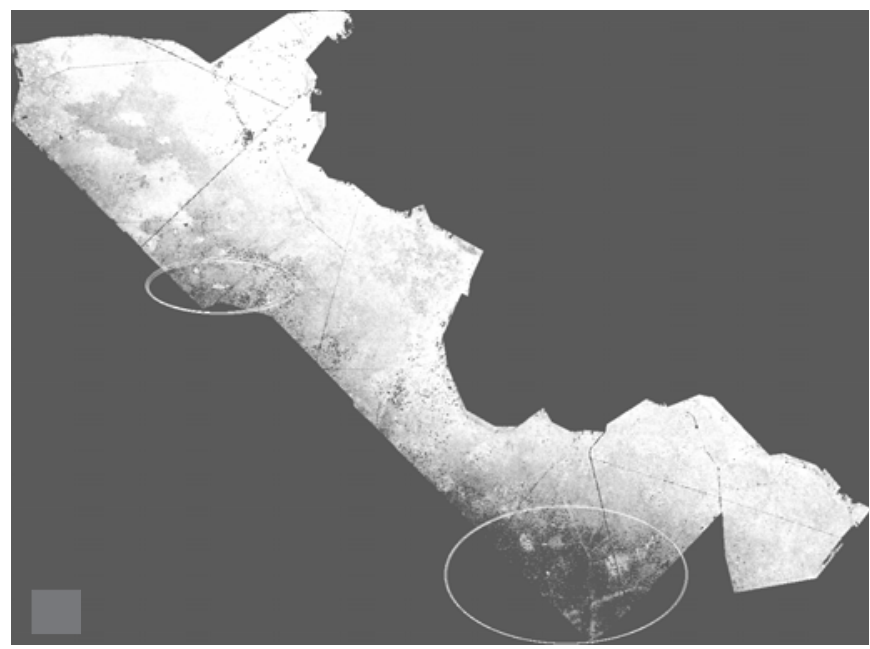

Obs.: Pontos pretos denotam "outliers". Os círculos na imagem (B) mostram as áreas com maior ocorrênci de "outliers"

Figura 2. Imagem NDVI da FLONA antes (A) e após (B) a normalização dos dados

Tabela 1. Reconstrução dos dados de radiância com base no número de fatores

\begin{tabular}{crrrr}
\hline \multirow{2}{*}{$\begin{array}{c}\text { Número } \\
\text { de }\end{array}$} & \multicolumn{4}{c}{ Pixels Reconstruídos } \\
\cline { 2 - 5 } Fatores & Total & $\begin{array}{c}\text { Com Base } \\
\text { no Fator }\end{array}$ & $\%$ & \% Acumulada \\
\hline 1 & 80761 & 80761,00 & 26,55 & 26,55 \\
2 & 277878 & 197117,00 & 64,81 & 91,36 \\
3 & 293682 & 15804,00 & 5,20 & 96,55 \\
mais de 3 & 304164 & 10482,00 & 3,45 & 100,00 \\
\hline
\end{tabular}

As relações entre as estimativas dos parâmetros estruturais da vegetação evidenciaram que cerca de $40 \%$ das variações no ABPSOM e na ABBSOM $\left(\mathrm{R}^{2}=0,39\right.$ e 0,40 , respectivamente $)$ podem ser explicados pelas estimativas da HTMED. Os valores relativamente baixos de $\mathrm{R}^{2}$ encontrados para as relações entre HTMED e ABPSOM e HTMED e ABBSOM, eram esperados, uma vez que a variável HTMED sofre influências da composição de espécies, da idade e, ainda, do índice de sítio (Husch et al., 1982, citados por Sousa \& Ponzoni, 1998); já as variáveis ABPSOM e ABBSOM são altamente correlacionadas entre si e, com a avaliação de uma delas, é possível explicar-se $95 \%$ da variação encontrada na outra. 


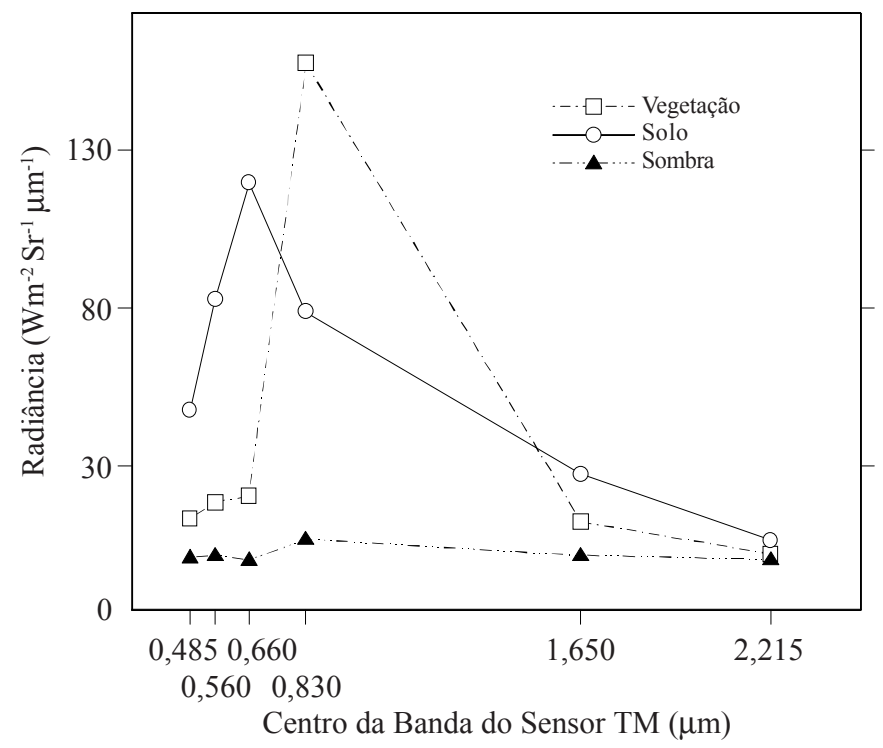

Figura 3. Curvas espectrais dos membros finais selecionados

Todas as variáveis espectrais apresentaram relacionamento positivo e significativo no nível de $1 \%$ de probabilidade, com as estimativas dos parâmetros estruturais HTMED, ABPSOM e ABBSOM (Tabela 2). As relações positivas entre as respostas espectrais no visível e as estimativas de parâmetros estruturais diretamente relacionados com as dimensões da copa das árvores, contradizem os resultados de trabalhos realizados em florestas naturais de coníferas (Franklin, 1986) e plantadas (Oza et al., 1989; Danson \& Curran, 1993). Franklin (1986) encontrou relações negativas entre área basal e a radiância no visível, registrada por um sensor multiespectral aerotransportável. Uma vez que o aumento da copa das árvores estava diretamente relacionado com o aumento da área basal, o autor atribuiu a redução da resposta espectral no visível ao aumento da sombra, causado pelo incremento da copa das árvores das parcelas que apresentavam maior área basal. A relação positiva

Tabela 2. Coeficientes de correlação (r) entre variáveis espectrais e estruturais

\begin{tabular}{lccc}
\hline \multicolumn{3}{c}{$\begin{array}{c}\text { Variável } \\
\text { Espectral }\end{array}$} & \multicolumn{3}{c}{ Variável Estrutural } \\
\cline { 2 - 4 } HTMED $^{8}$ & ABPSOM $^{9}$ & ABBSOM $^{10}$ \\
\hline Banda 1 & 0,48 & 0,35 & 0,38 \\
Banda 2 & 0,33 & 0,36 & 0,34 \\
Banda 3 & 0,46 & 0,49 & 0,50 \\
Banda 4 & 0,47 & 0,37 & 0,41 \\
Banda 5 & 0,49 & 0,51 & 0,52 \\
Banda 7 & 0,38 & 0,39 & 0,39 \\
FVV $^{1}$ & 0,51 & 0,47 & 0,50 \\
NDVI $^{2}$ & 0,49 & 0,47 & 0,50 \\
SR $^{3}$ & 0,60 & 0,56 & 0,58 \\
PVI $^{4}$ & 0,50 & 0,42 & 0,46 \\
GVI $^{5}$ & 0,53 & 0,45 & 0,49 \\
SAVI $^{6}$ & 0,49 & 0,47 & 0,50 \\
SI $^{7}$ & 0,60 & 0,54 & 0,57 \\
\hline
\end{tabular}

Fração da Vegetação Verde

Índice de Vegetação da Diferença Normalizada

Índice de Vegetação da Razão Simples

Índice Perpendicular de Vegetação

Índice de Verdor

Índice de Vegetação Ajustado para os Efeitos do Solo

Índice da Estrutura da Vegetação

Altura Média da Parcela

Área Basal à Altura do Peito

${ }^{10}$ Área Basal na Base encontrada para essas variáveis no presente trabalho, pode ser atribuída à baixa contribuição do componente sombra, com o aumento da contribuição da fração de vegetação, conforme indicado na Figura 4, na qual se verifica tendência de queda na fração de sombra, quando a fração de vegetação verde se eleva. A Figura 4 mostra que, para frações de vegetação entre 0,4 e 0,5 , as frações de sombra se situaram entre 0,47 e 0,52 , enquanto para frações de vegetação inferiores a 0,3 , as frações de sombra foram superiores a 0,57 . As diferenças na estrutura da vegetação e na composição florística (floresta de pinus ou florestas plantadas versus floresta tropical úmida, cerradão e cerrado) podem estar associadas às diferenças entre os resultados desses autores e os aqui apresentados.

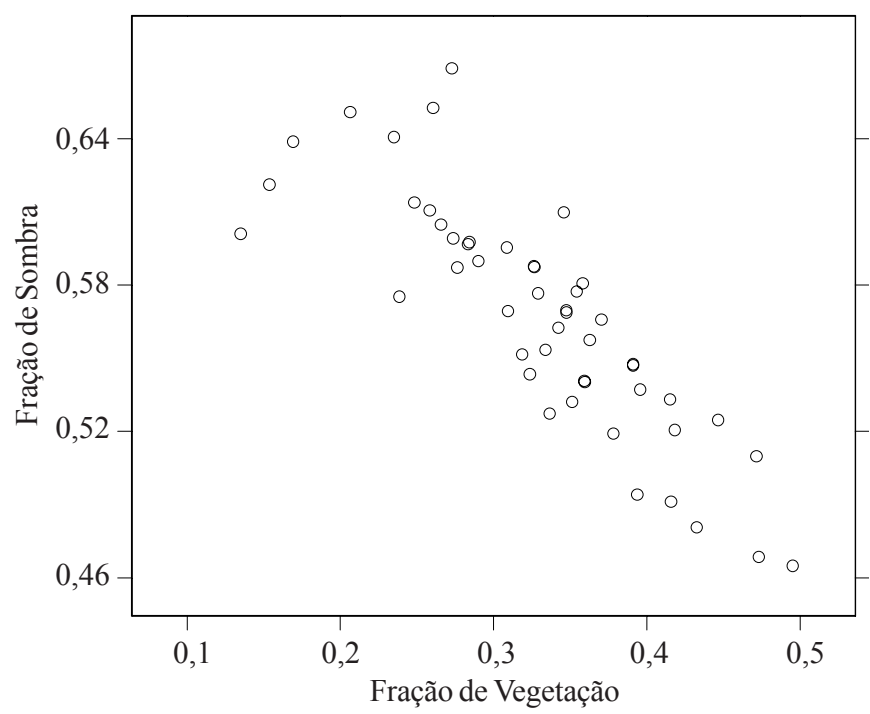

Figura 4. Diagrama de dispersão para as frações dos membros finais vegetação e sombra

As relações entre variáveis estruturais e espectrais foram, no geral, baixas, com valores para o coeficiente de correlação (r) variando entre 0,33 e 0,60 (Tabela 2). A variável estrutural que melhor se relacionou com as variáveis espectrais, foi HTMED. Resultados semelhantes foram encontrados por Jakubauskas \& Price (1997) ao tentarem determinar a relação entre altura de árvores maiores que 2,5 $\mathrm{m}$ com as 6 bandas do sensor TM, em vários modelos de regressão linear múltipla. Trabalhando em dados extrapolados para parcelas de 1 ha, esses autores encontraram que a variabilidade na altura média, no somatório da área basal, no valor total do índice de área foliar (LAI) e na idade das plantas, poderia ser explicada em 80, 63, 62 e $62 \%$ dos casos, respectivamente, pelo uso de variáveis espectrais.

As transformações espectrais (índices de vegetação e fração da vegetação proveniente da AME) apresentaram, em geral, maior relacionamento com as variáveis estruturais (Tabela 2). Esses resultados eram esperados, uma vez que as transformações são usadas para reduzir a influência de outros componentes presentes em um alvo, ao mesmo tempo em que realçam a resposta espectral da vegetação; além disso, transformações como os índices de vegetação, são mais sensíveis que a resposta espectral de bandas individuais às variações em estimativas de parâmetros da vegetação, como a biomassa e percentagem de cobertura vegetal (Asrar et al., 1984). 
Entre as transformações, as melhores relações entre variáveis estruturais e variáveis espectrais foram encontradas para o índice da razão simples (SR) e, para o índice estrutural da vegetação (SI). Esses resultados podem ser atribuídos à não saturação desses índices, quando comparados com as demais transformações espectrais, conforme apresentado na Figura 5; além da saturação, o fraco desempenho do SAVI pode estar, também, associado à baixa variabilidade dos solos da FLONA (Brasil, 1973). Para o caso da fração de vegetação proveniente da AME, a fraca relação obtida pode ser atribuída, ainda, às dificuldades inerentes à técnica na seleção dos melhores membros finais para a modelagem. A seleção de membros finais, com base no diagrama de dispersão das radiâncias para as diferentes bandas, pode ser confusa quando se tratam imagens com grandes dimensões (aproximadamente 334.000 pixels, no caso da FLONA).

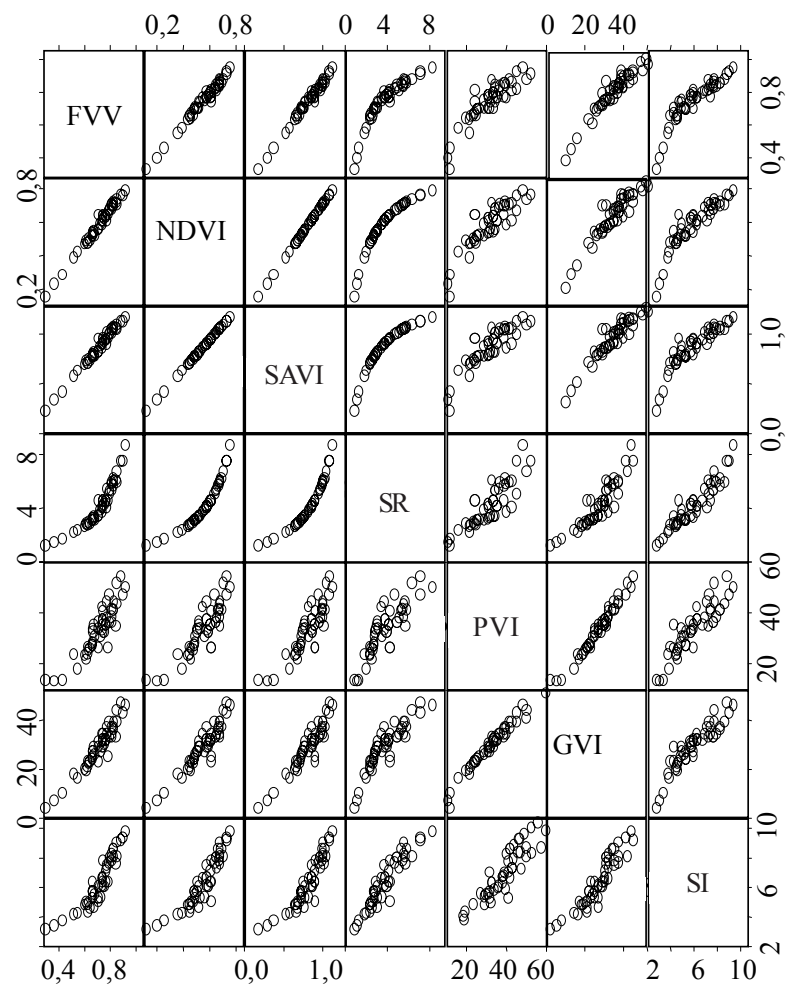

Figura 5. Diagrama de dispersão para os índices de vegetação

Uma solução para o problema poderia ser subdividir a imagem em segmentos e utilizar o modelo nesses segmentos menores, extraindo membros finais específicos para cada um dos segmentos. Esta solução, no entanto, é computacionalmente intensiva e pode não gerar resultados adequados para uma análise global das variações espaciais da estrutura da vegetação da FLONA. O melhor resultado encontrado para o SI pode ser atribuído à diversidade de tipologias de vegetação encontrada na FLONA. Esse índice tem sido utilizado com sucesso na separação de níveis de regeneração em florestas de pinheiros (Fiorella \& Ripple, 1993). As diferenças estruturais entre carrasco, cerrado, cerradão e mata úmida, podem causar respostas espectrais comparáveis àquelas obtidas em áreas com diferentes níveis de regeneração.

Trinta e seis porcento da variação em HTMED puderam ser explicados pelas equações que relacionaram essa variável aos índices SR e SI, enquanto que, para as variáveis ABPSOM e ABBSOM, esses mesmos índices explicaram em torno de 30\% das suas variações (Fig. 6). Considerando-se a complexidade da vegetação da FLONA, os ajustes encontrados são similares aos obtidos por outros autores em florestas cultivadas (Ponzoni \& Shimabukuro, 1998).

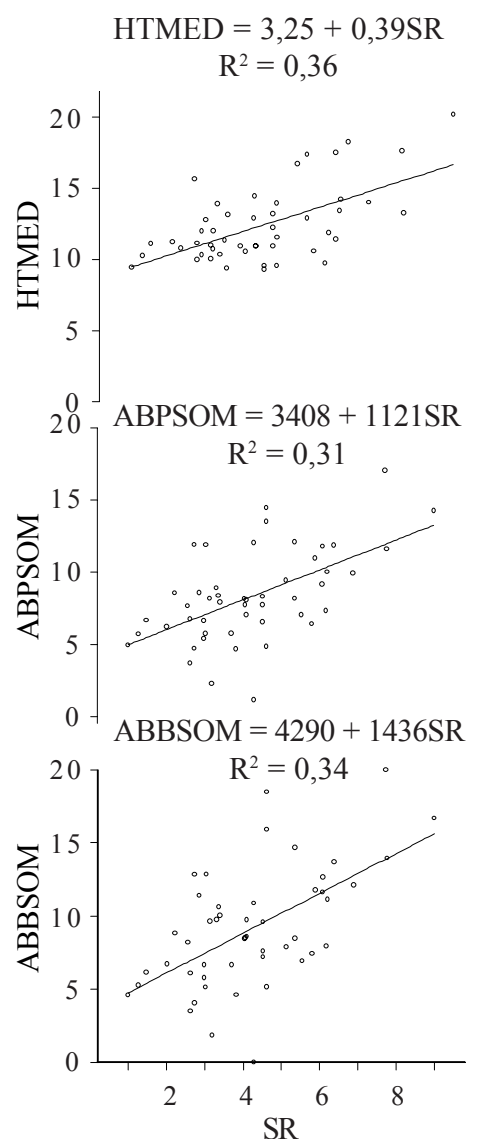

$$
\begin{gathered}
\mathrm{HTMED}=2,51+0,37 \mathrm{SI} \\
\mathrm{R}^{2}=0,36
\end{gathered}
$$

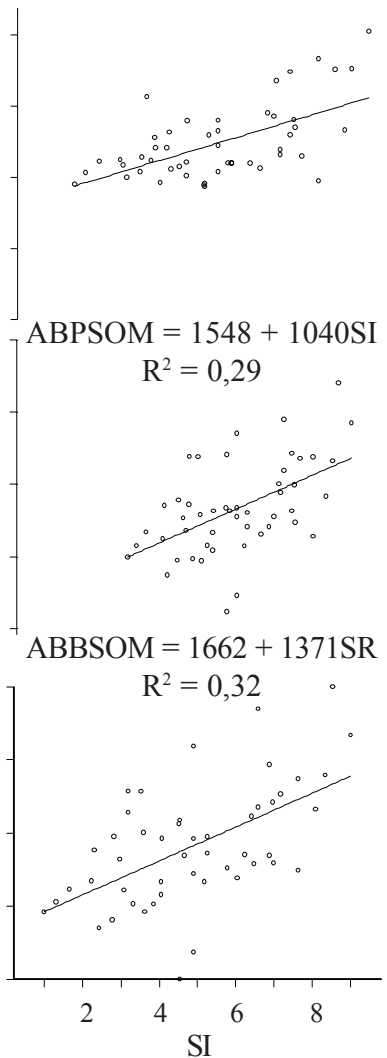

Figura 6. Variáveis estruturais $\operatorname{HTMED}(\mathrm{m}), \operatorname{ABPSOM}\left(\mathrm{m}^{2} \mathrm{ha}^{-1} 10^{3}\right)$ e $\operatorname{ABBSOM}\left(\mathrm{m}^{2} \mathrm{ha}^{-1} 10^{3}\right)$ em função dos índices de vegetação da razão simples (SR) e estrutural (SI)

\section{CONCLUSÕES}

1. A normalização dos dados espectrais para aplicação da análise de misturas espectrais indicou que cerca de $9 \%$ dos pixels da imagem da Floresta Nacional do Araripe eram 'outliers'.

2. Todas as variáveis espectrais (bandas isoladas, índices de vegetação e fração da vegetação obtida pelo modelo de análise de misturas) apresentaram correlação positiva e significativa a $1 \%$ de probabilidade com as estimativas dos parâmetros estruturais da altura média da parcela, da área basal à altura do peito e da área basal na base.

3. A variável estrutural que apresentou melhor correlação com as variáveis espectrais foi a altura média da parcela.

4. Quando comparadas às bandas isoladas, as transformações espectrais (índices de vegetação e fração da vegetação proveniente do modelo de análise de misturas), apresentaram, em geral, maior relacionamento com as variáveis estruturais.

5. O índice da razão simples e o índice estrutural da vegetação foram as transformações espectrais que melhor se relacionaram 
com as variáveis estruturais, explicando mais de $30 \%$ da variabilidade dos dados estruturais.

6. A fração da vegetação verde proveniente do modelo de análise de misturas não melhorou a predição de dados estruturais, quando comparada aos índices de vegetação testados neste trabalho.

\section{AGRADECIMENTOS}

A Ana Luiza da Silva, Bolsista de Iniciação Científica, pelos relatórios e monografias obtidos junto à Universidade Regional do Cariri (URCa) e à APA (Área de Proteção Ambiental) Chapada do Araripe.

\section{LITERATURA CITADA}

Asrar, G.; Funchs, M.; Kanemasu, E.T.; Hatfield, J.L. Estimating absorbed photosynthetic radiation and leaf area index from spectral reflectance in wheat. Agronomy Journal, Madison, v.76, p.300-306. 1984.

Brasil. Ministério da Agricultura. Departamento Nacional de Pesquisa Agropecuária. Divisão de Pesquisa Pedológica. Levantamento exploratório - reconhecimento de solos do estado do Ceará. Recife, SUDENE, 1973. 502p. Série Pedologia No. 16

Campelo, F.C.B.; Leal Júnior, G.; Silva, J.A. da; Campello, R.C.B. Avaliação dos recursos florestais da área de proteção ambiental, Chapada do Araripe. Crato: MMA, 2000. 49p.

Chavez Jr., P.S. Image-based atmospheric corrections - revisited and improved. Photogrammetric Engineering \& Remote Sensing, Bethesda, v.62, n.9, p.1025-1036. 1996.

Cibula, W.G. Use of topographic and climatological models in a geographical data base to improve Landsat MSS classification for Olympic National Park. Photogrammetric Engineering \& Remote Sensing, Bethesda, v.53, n.1, p.67-75, 1987.

Cohen, W.B.; Spies, T.A. Estimating structural attributes of douglas-fir/western hemlock forest stands from Landsat and Spot imagery. Remote Sensing of Environment, New York, v.41, p.1-17, 1992.

Danson, F.M.; Curran, P.J. Factors affecting the remotely sensed response of coniferous forest plantations. Remote Sensing of Environment, New York, v.43, p.55-65, 1993.

Fiorella, M.; Ripple, W.J. Analysis of conifer forest regeneration using Landsat Thematic Mapper data. Photogrammetric Engineering \& Remote Sensing, Bethesda, v.59, n.9, p.13831388, 1993.

Franklin, J. Thematic mapper analysis of coniferous forest structure and composition. International Journal of Remote Sensing, Abinjdon, v.7, n.10, p.1287-1301, 1986.
Huete, A.R. Soil influences in remotely sensed vegetationcanopy spectra. In: Asrar, G.(ed.). Theory and applications of optical remote sensing. New York: John Wiley \& Sons, 1989. cap. 4, p.107-141.

Huete, A.R.; Liu, H.Q.; Batchily, K.; van Leeuwen, W. A comparison of vegetation indices over global set of TM images for EOS-MODIS. Remote Sensing of Environment, New York, v.59, p.440-451, 1997.

Huete, A.R.; Warrick, A.R. Assessment of vegetation and soil water regimes in partial canopies with optical remotely sensed data. Remote Sensing of Environment, New York, v.32, p.155-167, 1990.

Jakubauskas, M.E.; Price, K.P. Empirical relationship between structural and spectral factors of Yellowstone lodgepole pine forests. Photogrammetric Engineering \& Remote Sensing, Bethesda, v.63, n.12, p.1375-1381, 1997.

Lopes, O.F.; Silva, F.H.B.B. Análise de dados climáticos da Chapada do Araripe, setor oriental, fazenda Ferreira Lopes. Crato: Informativo da Chapada, 1998. 6p.

Malinowski, E.R. Factor analysis in chemistry. New York: John Wiley \& Sons, 1991. 350p.

Mathsoft. S-Plus 4.0 Guide to Statistics. Seattle: MathSoft, Inc., 1997.650p

Oza, M.P.; Srivastava, V.K.; Pariswad, B.S.; Setty, R.V. Relationship between Landsat MSS data and forest tree parameters. International Journal of Remote Sensing, Abinjdon, v.10, n.11, p.1813-1819. 1989.

Ponzoni, F.J.; Shimabukuro, Y.E. Relação entre índice de vegetação (NDVI e SAVI) e parâmetros biométricos de plantios de Eucalyptus urophylla e Eucalyptus camaldulensis em Cachoeira do Manteiga MG. Revista Árvore, Viçosa, v.22, n.3, p.357-366, 1998.

Smith, M.O.; Ustin, S.L.; Adams, J.B.; Gillespie, A.R. Vegetation in Deserts: I. A regional measure of abundance from multispectral images. Remote Sensing of Environment, New York, v.31, p.1-26, 1990.

Souza, C.L.; Ponzoni, F.J. Relação entre índice de área foliar, estimado através de sensoriamento remoto, e parâmetros dendrométricos em floresta implantada de Pinus spp. In: Simpósio Brasileiro de Sensoriamento Remoto, 9, 1998, Santos. Anais... Santos: INPE/SELPER, 1998. CD-Rom

Thome, K.; Markham, B.; Barker, J.; Slater, P.; Biggar, S. Radiometric calibration of Landsat. Photogrammetric Engineering \& Remote Sensing, Bethesda, v.63, n.7, p.853-858, 1997.

Todd, S.W.; Hoffer, R.M. Responses of spectral indices to variations in vegetation cover and soil background. Photogrammetric Engineering \& Remote Sensing, Bethesda, v.64, n.9, p.915-921, 1998.

Toniolo, E.R.; Kazmierczak, M.L. Mapeamento da Floresta Nacional do Araripe (Flona-Araripe). Fortaleza: IBAMA, 1998. 12p. 